\title{
Male Infertility and Assisted Reproductive Technology: Lessons from the IVF
}

\author{
Sandro C. Esteves* and Danielle T. Schneider
}

ANDROFERT, Andrology and Human Reproduction Clinic, Campinas, São Paulo, Brazil

\begin{abstract}
Development in ART has been remarkable in the last decades. Several medical conditions affecting the male fertility status are now treated by ART, especially by the combination of in vitro fertilization and intracytoplasmic sperm injection. ICSI is now the treatment of choice of most untreatable causes of male infertility, including immunologic infertility, severe oligozoospermia and azoospermia. Sperm retrieval techniques have been optimized and microsurgery offers the possibility of collecting testicular spermatozoa even in the most difficult cases of non-obstructive azoospermia. Nonetheless, the reproductive potential of sperm from men with defective spermatogenesis is decreased in ART and such treatment modalities may carry an increased risk of transmitting genetic and epigenetic defect to the embryo. Efforts should be made to improve the male health status prior to embarking on ART because current evidence suggests that fertility optimization may improve treatment outcomes. Moreover, laboratory management of male infertility cases requires special attention. Spermatozoa collected from men with severely impaired spermatogenesis are often compromised and fragile. Adherence to state of the art laboratory techniques and quality control are recommended to avoid jeopardizing sperm fertilizing potential and the chances of achieving a live birth. In this study, we present and critically review our 10-year experience in the management of severe male factor infertility using ART.
\end{abstract}

Keywords: Male infertility, assisted reproductive technology, intracytoplasmic sperm injection, azoospermia, oligozoospermia, antisperm antibodies, laboratory techniques.

\section{INTRODUCTION}

In a group of 2,383 male infertility patients attending our tertiary center for male reproduction, potentially surgically or medically correctable conditions were identified in $48.4 \%$ of the individuals. The other half comprised candidates for assisted reproduction, particularly assisted reproductive technologies (ART) involving in vitro fertilization (IVF) coupled to intracytoplasmic sperm injection (ICSI) (Table 1). In this review, we discuss our 10-year experience in the management of severe male factor infertility using ART.

\section{THE ROLE OF THE MALE PARTNER}

Infertility is a common clinical problem that affects approximately $8 \%$ of the male population in reproductive age [1]. It is defined as the failure to conceive after one year of regular intercourse without contraception, and traditionally, investigation starts at this point. However, it is recommended that investigation is initiated earlier whenever risk factors are present since longer infertility duration relates to smaller chances of success regardless of the treatment strategy adopted [2,3]. Determining the couple's fertility potential is a complex process that involves both male and female partners. The cause of infertility may or not be identified and can involve one or both partners.

The cornerstones for the evaluation of a subfertile man include a comprehensive history, physical examination and

*Address correspondence to this author at the ANDROFERT - Andrology and Human Reproduction Clinic, Av. Dr. Heitor Penteado, 1464, 13075460, Campinas, São Paulo, Brazil; Tel: 55193295-8877; Fax: 551932946992; E-mail: s.esteves@androfert.com.br
Table 1. Distribution of Diagnostic Categories in a Group of 2,383 Infertile Men Attending a Male Infertility Clinic

\begin{tabular}{|c|c|c|}
\hline Category & $\mathbf{N}$ & $\mathbf{\%}$ \\
\hline \hline Varicocele & 629 & 26.4 \\
\hline Infectious & 72 & 3.0 \\
\hline Hormonal & 54 & 2.3 \\
\hline Ejaculatory dysfunction & 28 & 1.2 \\
\hline Systemic diseases & 11 & 0.4 \\
\hline Idiopathic & 289 & 12.1 \\
\hline Immunologic & 54 & 2.3 \\
\hline Obstruction & 359 & 15.1 \\
\hline Cancer & 11 & 0.5 \\
\hline Cryptorchidism & 342 & 14.3 \\
\hline Genetic & 189 & 7.9 \\
\hline Testicular failure & 345 & 14.5 \\
\hline TOTAL & 2,383 & 100.0 \\
\hline
\end{tabular}

multiple semen analyses. Advanced paternal age, inadequate diet intake, drug abuse, pesticide environmental exposure, tobacco use, varicocele, medical diseases, hyperthermia, air pollution, genital inflammation or infectious diseases, history of urogenital surgery, and cancer can be cited as possible causes or contributing factors of male subfertility which may be reversed or treated in several cases [4-9]. The seminal 
analysis is the basis of the laboratory evaluation; it provides information on the functional status of the germinative line, epididymis and accessory sexual glands. An ejaculated semen sample can harbor varying degrees of sperm abnormalities ranging from absence to severe alterations in sperm parameters. Beyond the conventional semen analysis, additional tests have been developed in order to identify functional disorders and other spermatic abnormalities that are not addressed in routine semen evaluation. Such tests include assessment of antisperm antibodies and sperm DNA integrity $[10,11]$. Levels of antisperm anti-bodies and sperm DNA integrity are associated with the male fertility potential $[12,13]$. An endocrine evaluation is also part of the initial male workup. Genetic testing, such as karyotyping and testing for microdeletions of the $\mathrm{Y}$ chromosome or cystic fibrosis gene mutation screening, is indicated in selected cases of azoospermia and severe oligozoospermia, particularly prior to assisted conception.

\section{ASSISTED REPRODUCTIVE TECHNOLOGY FOR THE MALE}

Treatments or procedures that involve in vitro handling of human oocytes and sperm or embryos for the purpose of establishing a pregnancy are included in the ART category. In the context of male infertility, ART procedures include in vitro fertilization (IVF), IVF with intracytoplasmic sperm injection (ICSI) and sperm and embryo cryopreservation. ART does not include assisted insemination (artificial insemination) using sperm from either a woman's partner or sperm donor [14]. Two major breakthroughs occurred in the area of male infertility in the last years. The first was the development of intracytoplasmic sperm injection (ICSI) for the treatment of male factor infertility due to severely abnormal semen quality [15]. The second was the extension of ICSI to azoospermic males and the demonstration that spermatozoa derived from either the epididymis or the testis were capable of normal fertilization and pregnancy $[16,17]$.

\section{INTRACYTOPLASMIC SPERM INJECTION TREAT- MENT STEPS}

\section{Controlled Ovarian Stimulation (COS)}

In our center, COS for ART is performed using either the long pituitary desensitization gonadotropin-releasing hormone agonist (GnRH-a) or the $\mathrm{GnRH}$ antagonist protocol [18]. For ovarian stimulation, initial daily doses of 150-375 IU of gonadotropins (human menopausal gonadotropin [hMG], high-purified hMG or recombinant human follicle stimulating hormone $[\mathrm{r}-\mathrm{hFSH}])$ are used. The initial dose of gonadotropin is determined by the treating physician taking into account female characteristics such as age, body mass index, serum FSH on day 2 or 3 of the menstrual cycle, baseline ovarian volume on transvaginal ultrasound (TVUS), and number of pre-antral follicles between days 2 and 3 of the menstrual cycle. For cycles involving long pituitary desensitization, treatment is initiated with $400 \mu \mathrm{g}$ daily intranasal administration of gonadotropin-releasing hormone agonist (nafarelin acetate), starting on the twenty-first day of the menstrual cycle and is maintained until the day before human chorionic gonadotropin (hCG) administration. Ova- rian stimulation commences only after confirming pituitary down-regulation by both serum estradiol levels $<50 \mathrm{pg} / \mathrm{mL}$ and the absence of ovarian follicles $>10 \mathrm{~mm}$ in diameter on TVUS. For cycles involving GnRH antagonist, ovarian stimulation is initiated either on day 2 or 3 of the menstrual cycle after confirmation of the absence of ovarian follicles $>10 \mathrm{~mm}$ on TVUS. A daily dose of $0.25 \mathrm{mg}$ subcutaneous administration of GnRH antagonist is started when the lead follicle reaches $14 \mathrm{~mm}$ or $\geq 5$ follicles are between $11-13 \mathrm{~mm}$ (usually between days 5 and 7 but no later than day 8 of stimulation) and is continued up to and including the day of hCG administration. Ultrasound assessment between the sixth and eighth days of stimulation is performed to determine if gonadotropin dose adjustments are required. Human chorionic gonadotropin (hCG) is administered when two or more ovarian follicles reach a mean diameter of $18 \mathrm{~mm}$.

\section{Oocyte Retrieval, Sperm Processing, and ICSI}

Oocyte retrieval is performed under intravenous sedation and guided by TVUS, 34-36 hours after hCG administration. Cumulus-corona-oocytes complexes are denuded, classified according to nuclear maturity and are maintained in culture until sperm microinjection. Ejaculated spermatozoa are processed by discontinuous colloidal gradient or swim-up techniques [19]. In the cases of azoospermia, either percutaneous testicular or epididymal sperm aspirations, or microsurgical testicular sperm extraction are performed for sperm retrieval [20]. Selection and immobilization of the spermatozoon, and microinjections, are performed under a $400 \mathrm{X}$ magnification [21]. The injected oocytes are transferred to a closed culture system and incubated for $16-18$ hours at $37^{\circ} \mathrm{C}$ and $5.5 \% \mathrm{CO}_{2}$, until confirmation of fertilization. Fertilization was considered normal when oocytes with two pronuclei are seen.

\section{Embryo Culture, Cleavage Check, Transfer, and Luteal Support}

Fertilized oocytes are maintained in culture until transfer of the embryos to the uterine cavity on the third or fifth day of embryo culture [22]. Embryo quality is scored daily, and the number, symmetry, and expansion of the blastomeres, multinucleation, anomalies of the zona pellucida, the rate of cytoplasmic fragmentation and blastocyst formation are recorded. The embryos are classified as top quality when they had three to four symmetrical blastomeres on the second day of culture and seven to eigth symmetrical blastomeres on the third day, with no multinucleation, grade I (no fragmentation) or grade II fragmentation (up to $20 \%$ of the perivitelline space with fragments), and no abnormalities in the zona pellucida [23]. One to four embryos are transferred to the uterine cavity depending on the couples' clinical profile and regulatory guidelines. Abdominal ultrasound guidance is used during all embryo transfers, which are performed three to five days post-oocyte retrieval. Luteal phase support with once-daily application of progesterone gel is initiated in the same day of oocyte retrieval. Embryos that are not transferred are cryopreserved for future use.

Oocyte collection, micromanipulation of gametes, embryo culture, and the transfer of embryos to the uterine 
cavity are carried out under ISO 6 , ISO 5 , and ISO 7 cleanroom environments, respectively (ANDROFERTVECO, Brazil) [24, 25].

\section{INTRACYTOPLASMIC TECHNICAL ASPECTS}

\section{SPERM INJECTION}

\section{Collection of Ejaculated Specimens: Effect of Stress on Semen Quality}

It has been shown that infertility-related stress is significantly increased in couples undergoing ART treatments [26]. Although men tend to report less sexual stress and anxiety than women, a strong linkage between anxiety and sexual stress may be observed in men with severe male factor infertility [27]. In order to examine the impact of anxiety on semen quality, we conducted a study involving 160 men with severe male factor infertility enrolled in our ART program [28]. Ejaculated semen specimens were obtained at two different occasions using identical ejaculatory abstinence intervals: i) at the time of male infertility workup, and ii) at the time of oocyte collection for sperm processing and ICSI. We noted that sperm concentration and progressive motility were significantly lower in semen samples collected on the day of oocyte retrieval as compared to specimens provided for previous seminal analysis. Moreover, approximately $20 \%$ of men with severe oligozoospermia on diagnostic semen analysis produced an azoospermic specimen at the time of ICSI, requiring subsequent collection attempts or testicular sperm retrieval. A qualitative questionnaire analysis showed that the proportion of men experiencing anxiety was significantly higher at the day of female partners' oocyte collection than on previous semen analysis for diagnostic purposes. Our results suggested that acute psychological stress can impair semen quality of men with severe male factor infertility enrolled in ART. We advise our patients with severe oligozoospermia to cryobank their specimens prior to ART to avoid the risk of obtaining very poor semen specimens at the time of the ICSI cycle.

\section{Optimal Time Interval for Microinjection}

In ART treatment cycles involving severe male factor infertility, particularly in non-obstructive azoospermia, the time frame between oocyte retrieval and microinjection may be long due to technical difficulties for testicular tissue processing and sperm procurement. The oocyte lifespan is, however, limited, and it has been suggested that the oocyte retains its optimal developmental capacity if insemination is kept within a window of 10 hours from hCG administration [29-31]. We evaluated the impact of the time interval between hCG administration and sperm microinjection on fertilization and embryo development in severe male factor infertility ART [32]. A total of 550 ICSI cycles involving 4,334 oocytes were reviewed. The sperm sources for microinjection were ejaculate $(n=364)$, epididymis $(n=30)$ and testicle $(n=75)$. Injected oocytes were grouped according to the time elapsed between hCG administration and microinjections. The case assignment was random and based only by workload. Our findings revealed that oocytes microinjected either before 38 or after 43 hours, in relation to hCG administration, exhibited significant lower 2PN fertilization rates and embryo development than those injected within the time interval of 38-42 hours (Table 2). A significant proportion of oocytes were injected with testicular spermatozoa in the group of $>43$-hour time interval from hCG to microinjections. We suspected that the use of testicular spermatozoa from non-obstructed azoospermic men justified our findings of decreased fertilization and embryo development in this subgroup. However, reduced fertilization and embryo development were still observed, at marginally significant levels, in the microinjection interval subgroup of longer than 43 hours after excluding the oocytes injected with testicular sperm. Oocytes matured in vitro and injected on the next day also showed significant lower fertilization and embryo development. We concluded that the optimal time interval for microinjection in cases of severe male factor infertility is 38-42 hours after hCG administration. One should expect lower fertilization and embryo development with microinjections performed $>43 \mathrm{~h}$ after hCG, particularly in the cases of non-obstructive azoospermia. It is advisable to time sperm retrieval procedures at early hours on the day of ICSI, or at the previous day, in cases involving testicular sperm collection from men with non-obstructive azoospermia. This strategy allows microinjections to take place at the optimal injection window, thus avoiding jeopardizing ART outcomes.

\section{Optical Magnification for Sperm Selection and ICSI}

In a prospective and randomized clinical study, we examined the effect of optical magnification for sperm selection and microinjection on ICSI outcomes of 120 infertile couples

Table 2. ICSI Outcomes According to the Time Elapsed between hCG Administration and Microinjections. (Adapted from Fertil Steril. 86, Suppl, Schneider DT, et al. Optimal time interval for intracytoplasmic sperm injection after human chorionic gonadotropin administration in severe male factor infertility, page S155, Copyright 2006, with permission from Elsevier)

\begin{tabular}{|c|c|c|c|c|c|}
\hline $\begin{array}{c}\text { Time between hCG administration } \\
\text { and microinjection }\end{array}$ & $\begin{array}{c}\text { Oocytes } \\
\text { injected (n) }\end{array}$ & $\begin{array}{c}\text { \% Normal 2PN } \\
\text { fertilization }\end{array}$ & $\begin{array}{c}\text { \% Abnormal } \\
\text { fertilization }\end{array}$ & $\begin{array}{c}\text { \% Top quality } \\
\text { embryos on Day 3* }\end{array}$ & $\begin{array}{c}\text { \% Oocytes injected with } \\
\text { testicular sperm (\%) }\end{array}$ \\
\hline \hline Group 1 (<38h) & 60 & $63[43-86]$ & 0 & $7[0-28]$ & 0 \\
\hline Group 2 (38-43h) & 3014 & $73[50-100]$ & $0[0-14]$ & $50[0-75]$ & 26 \\
\hline Group 3 (>43h) & 1079 & $56[38-86]$ & $0[0-20]$ & $33[0-67]$ & 37 \\
\hline Group 4 (day after) & 181 & $44[0-100]$ & 0 & NA & 18 \\
\hline P-value & - & $\begin{array}{c}\text { G2 vs. G3: } \mathrm{p}<0.01 ; \\
\text { G1-3 vs. G4: } \mathrm{p}<0.01\end{array}$ & NS & $\begin{array}{c}\text { G1 vs. G2: } \mathrm{p}<0.01 ; \\
\text { G2 vs. G3: } \mathrm{p}=0.02\end{array}$ & G3 vs. G2/G4: $\mathrm{p}<0.01$ \\
\hline
\end{tabular}

*7-9 blastomeres of similar size, and grades I or II cytoplasm fragmentation on the day of embryo transfer (day 3 ). Values are expressed as median and $25 \%-75 \%$ percentiles. Kruskal-Wallis statistic was used for comparisons among groups. NS: not significant. 
[21]. Patients were randomized according to the optical magnification power used for sperm selection and microinjection, i.e., $200 \mathrm{X}$ or $400 \mathrm{X}$. Sperm with normal morphology were selected for injection, whenever possible, and sperm with large vacuoles and cytoplasmic droplets were excluded in the 400X magnification group. Microinjections were performed with the polar body at 6 or 7 o'clock position in the 200X group, and with the polar body at different positions in the 400X group to allow microinjections to take place at the vegetal oocyte pole. The normal 2PN fertilization rate was significantly increased in the $400 \mathrm{X}$ group $(74.1 \% \pm 23.8 \%)$ compared to the $200 \mathrm{X}$ group $(57.4 \%$ $\pm 30.2 \%, p=0.001$ ), while the percentage of abnormally fertilized oocytes was significantly reduced in the former $(1.4 \% \pm 4.7 \%$ vs. $11.4 \% \pm 17.5 \%, \mathrm{p}<0.0001)$. Our results also showed that the proportion of top quality embryos available for transfer and the clinical pregnancy rate were significantly higher in the $400 \mathrm{X}$ group $(54.6 \% \pm 34.9 \%$ and $38.8 \%)$ compared to the $200 \mathrm{X}$ group $(27.8 \% \pm 28.3 \%$ and $24.9 \%(\mathrm{p}<0.001)$. Based on these data, it is our routine to use 400X magnification for sperm selection and microinjection in ICSI cycles. Although intracytoplasmic morphologicallyselected sperm injection (IMSI) using high power magnification has been advocated to yield better ART outcomes [33], we found that it is very easy to exclude sperm with large vacuoles and/or with cytoplasmic droplets at $400 \mathrm{X}$ magnification, as shown by others [34]. At 400X magnification, it is also possible to identify and to perform microinjections at the oocyte vegetal pole to minimize the risk of damaging the oocyte meiotic spindle.

\section{Air Quality in the IVF Laboratory: Impact on ART Treatment Results}

Airborne concentrations of toxics volatile organic compounds (VOC), chemical contaminants and particles may impair embryo development and pregnancy rates [35-37]. Volatile organic compounds are produced by industries, vehicles, heating exhausts, cleaning products, furniture and others. Considering these facts, some companies have designed filtration units to clean the air of the in vitro fertilization (IVF) laboratory and to decrease contamination with varying efficiency levels [38]. Cleanrooms are contaminated-free environments useful for certain critical applications in bioengineering, pharmaceutical and computer industries. We have studied the impact of high standard measures for air quality control inside the IVF laboratory and adjacent areas on ART outcomes [24, 25]. In one study, we evaluated 399 consecutive ICSI cycles in which the primary indication for treatment was severe male factor infertility. ART was performed inside two different Cleanroom IVF laboratories classified in accordance to the International Standard Organization (ISO) 14.644-1 [25]. IVF laboratory ISO type 6 was equipped with a positive pressure high efficiency particles air filtration (HEPA) system (VECO, Brazil) and a free-standing unit to filter and remove VOC (Coda Tower, GenX, USA). IVF lab ISO type 5 was built with proper engineering and material selection. It included a dedicated air handling system equipped with HEPA and carbon-impregnated filters, located in a separate room, to filter and remove particles and VOC from the IVF laboratory and adjacent areas such as the oocyte retrieval and embryo transfer rooms (ANDROFERT-VECO, Brazil). A positive pressure differential was maintained from the ISO 5 IVF laboratory to the oocyte retrieval (ISO 6 Cleanroom) and the embryo transfer rooms (ISO 7 Cleanroom). Incubators in both labs were equipped with in-line carbon impregnated HEPA filters for carbon dioxide from gas cylinders (GenX, USA). In this series, female age, duration of ovarian stimulation, amount of gonadotropin required per cycle, number of oocytes retrieved and proportion of ICSI cycles involving microinjection of epididymal or testicular spermatozoa were not statistically different between groups. We found that cleavage, embryo quality and clinical pregnancy rates were significantly increased, while miscarriage rates were reduced, in the group of ICSI cycles performed inside the ISO type 5 IVF laboratory as compared to the ISO type 6 laboratory (Table 3). We concluded that air quality control in the IVF laboratory and critical areas may impact on embryo development and pregnancy results. Our observations are consistent with an association between the presence of air contaminants in the IVF laboratory and ART outcome.

\section{ANTISPERM ANTIBODIES AND ICSI RESULTS}

Antisperm antibodies (ASA) are found in 3\% to $12 \%$ of men who undergo evaluation for infertility [12]. IgA and IgG immunoglobulins are of clinical importance because these are the subclasses that have been demonstrated in the ejacu-

Table 3. Comparison of ICSI outcomes between cycles performed in the ISO 6 and ISO 5 IVF laboratories. (Adapted from Fertil Steril. 86, Suppl, Esteves SC, et al. Comparison between International Standard Organization (ISO) type 5 and type 6 cleanrooms combined with volatile organic compounds filtration system for micromanipulation and embryo culture in severe male factor infertility, pages 353-4, Copyright 2006, with permission from Elsevier)

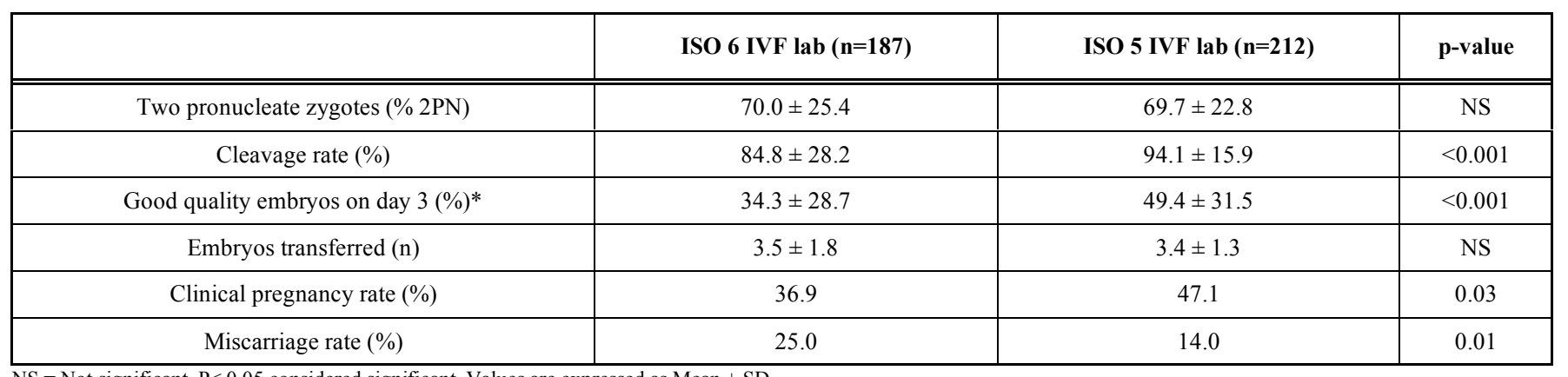

NS $=$ Not significant. $\mathrm{P}<0.05$ considered significant. Values are expressed as Mean $\pm \mathrm{SD}$

*Good quality embryos: Embryos with 7-8 blastomeres on culture day 3, and cytoplasmic fragmentation $\leq 20 \%$. 
lates of men with antisperm autoimmunity. The formation of antisperm antibodies (ASA) may be secondary to the rupture of the blood-testis barrier. Obstruction, inflammation and trauma of the genital tract, as well as cryptorchidism, varicoceles and vasectomy have been associated with elevated levels of ASA [39]. ASA may cause sperm agglutination and/or immobilization, thus decreasing the fertilizing potential by impairing progression through the female genital tract and/or by interfering with the fertilization process. ASA may also impair sperm capacitation and acrosome reaction, thus affecting sperm-oocyte interaction [40, 41]. Decreased fertilization and cleavage rates are expected when ASA bound sperm are used in conventional in vitro fertilization (IVF) or in intrauterine insemination [42-44]. Fertilization rates tended to decrease as the amount of antibody increased in the direct immunobead test (IBT) [45], and very low fertilization rates have been observed when $>70 \%$ of inseminated spermatozoa were coated with ASA. Once fertilization had occurred, the pregnancy rate was not affected by the severity of immunological factors [46].

Alternatives to overcome the deleterious effects of ASA, such as medication [47-49], sperm washing combined with intrauterine insemination $[47,50]$ and in vitro fertilization [42-45, 51-53] have been proposed. The effectiveness of these techniques in recovering antibodies-free spermatozoa are conflicting; most reports show limited success due to the difficulty of eluting the sperm cell surface by any washing method [54, 55]. In a previous report, we demonstrated that sperm processing may be helpful in eluting antibodies-bound to sperm. We studied 48 men with varying levels of ASA in the semen, as determined by the immunobeads binding test (IBT), and found that the population of antibodies-free spermatozoa was increased by $29 \%$ after discontinuous colloidal gradient centrifugation. We also noted that sperm washing was ineffective to remove ASA in approximately $30 \%$ of the cases, and advise that the potential benefit of this strategy has to be tested individually [56].
Microinjection of the compromised spermatozoa into the oocyte cytoplasm (ICSI) bypasses sperm-oocyte membrane interaction, and ICSI has been shown to increase fertilization when compared to conventional IVF in cases of male immunologic infertility. Nagy et al. analyzed the outcome of ICSI in 37 men with a proportion of antisperm antibodybound spermatozoa of $80 \%$ or higher [57]. They concluded that fertilization, cleavage and pregnancy rates after ICSI were not influenced by the percentage of ASA-bound spermatozoa, by the dominant type of antibodies present, or by the location of ASA on the spermatozoa. However, embryo quality was lower in the ASA-positive group. In another study, similar results have been observed but a higher rate of first trimester pregnancy loss in the ASApositive group has occurred [58]. Clarke et al. [59] and Check et al. [60] studied 39 patients with a strong positivity on IBT $(>80 \%)$ and 93 patients with various degrees of autoantibodies, respectively. They found that fertilization and pregnancy rates were comparable between different levels of ASA on sperm. In order to re-examine data of ICSI in the light of the above mentioned reports, we analyzed ICSI outcome in large cohort of 351 patients at four different levels of ASA in the semen, as determined by direct immunobeads testing [10]. Our findings confirmed that fertilization, cleavage and pregnancy rates after ICSI were not influenced by the ASA levels on sperm (Table 4). Additionally, we have neither observed the negative impact on embryo quality nor the increase in pregnancy loss in the sperm antibody-positive patients, as reported by others. We also analyzed whether cleavage velocity is altered by ASA levels, and our results indicated that, irrespective of ASA levels, cleavage velocity was not altered by antisperm antibody-bound to spermatozoa. In our study, we also compared ICSI outcomes between patients with ASA positivity and a group of patients in which ICSI was indicated for other reasons. Our findings demonstrated that fertilization, embryo development, pregnancy success and

Table 4. Comparative Analysis of the Outcomes of 351 ICSI Cycles Subdivided into 4 Groups According to the Percentage of ASA as Tested by Direct Immunobeads Assay, and 349 ICSI Cycles from Oligo/Asthenozoospermic Men in which ASA Testing has not been Performed (Adapted from International Braz J Urol 34, Esteves SC, et al. Influence of Antisperm Antibodies in the Semen on Intracytoplasmic Sperm Injection, pages 795-802, Copyright 2007, with permission)

\begin{tabular}{|c|c|c|c|c|c|c|}
\hline & $\begin{array}{c}\text { Group } 1(n=194) \\
0-10 \% \text { ASA }\end{array}$ & $\begin{array}{c}\text { Group } 2(n=107) \\
11-20 \% \text { ASA }\end{array}$ & $\begin{array}{c}\text { Group } 3(n=33) \\
21-50 \% \text { ASA }\end{array}$ & $\begin{array}{c}\text { Group } 4(n=17) \\
51-100 \% \text { ASA }\end{array}$ & $\begin{array}{c}\text { Group } 5(\mathrm{~N}=349) \text { ASA } \\
\text { not determined }\end{array}$ & p-value \\
\hline $\begin{array}{l}\text { Sperm count } \\
\left(\mathrm{X} 10^{6} / \mathrm{mL}\right)\end{array}$ & $\begin{array}{c}33.0 \\
{[10.7-75.7]^{\mathrm{a}}}\end{array}$ & $\begin{array}{c}44.7 \\
{[9.2-65.0]^{\mathrm{b}}}\end{array}$ & $\begin{array}{c}58.0 \\
{[24.0-95.4]^{\mathrm{c}}}\end{array}$ & $\begin{array}{c}13.3 \\
{[12.9-92.0]^{\mathrm{d}}}\end{array}$ & $\begin{array}{c}2.7 \\
{[0.5-12.0]^{\mathrm{e}}}\end{array}$ & $\begin{array}{c}\mathrm{a}, \mathrm{b}, \mathrm{c}, \mathrm{d} v s . \mathrm{e}: \\
\mathrm{p}<0.01\end{array}$ \\
\hline $\begin{array}{c}\% 2 \mathrm{PN} \\
\text { fertilization }\end{array}$ & $\begin{array}{c}80.0 \\
{[66.7-100.0]}\end{array}$ & $\begin{array}{c}75.0 \\
{[60.0-90.0]}\end{array}$ & $\begin{array}{c}75.0 \\
{[66.7-83.3]}\end{array}$ & $\begin{array}{c}82.4 \\
{[70.0-100.0]}\end{array}$ & $\begin{array}{c}71.4 \\
{[50.0-87.5]}\end{array}$ & NS \\
\hline $\begin{array}{l}\% \text { Slow cleavage } \\
\text { velocity }\end{array}$ & $\begin{array}{c}45.5 \\
{[25.0-66.7]}\end{array}$ & $\begin{array}{c}50.0 \\
{[16.7-63.6]}\end{array}$ & $\begin{array}{c}36.7 \\
{[0.0-65.5]}\end{array}$ & $\begin{array}{c}42.9 \\
{[16.7-71.4]}\end{array}$ & $\begin{array}{c}42.8 \\
{[16.6-66.6]}\end{array}$ & NS \\
\hline $\begin{array}{l}\% \text { Good quality } \\
\text { embryos* }\end{array}$ & $\begin{array}{c}50.0 \\
{[27.3-66.7]}\end{array}$ & $\begin{array}{c}50.0 \\
{[25.0-71.4]}\end{array}$ & $\begin{array}{c}39.2 \\
{[18.3-66.7]}\end{array}$ & $\begin{array}{c}57.1 \\
{[28.6-83.3]}\end{array}$ & $\begin{array}{c}40.0 \\
{[20.0-61.5]}\end{array}$ & NS \\
\hline
\end{tabular}

* 7-9 blastomeres of similar size, and grades I or II cytoplasm fragmentation on the day of embryo transfer (day 3). Kruskal-Wallis statistic was used for comparisons among groups. Values are expressed as median [25-75 percentiles]. NS: not significant. 
miscarriage rates after ICSI in men exhibiting varying levels of autoimmunity against spermatozoa were within the same range as our population of ICSI patients with severely abnormal seminal parameters. We speculate that ASA may become inactive within the ooplasm after microinjection, or that a segregation process may take place during the first cleavage divisions, similar to the inactivation and segregation processes that also occur with the acrosome and sperm tail after microinjection.

\section{IMPACT OF SPERM DEFECT SEVERITY ON ART}

Traditionally, male factor infertility is described in terms of abnormal sperm concentration (oligozoospermia), impaired sperm motility (asthenozoospermia) or morphology (teratozoospermia). In several cases, a combination of two or more of these sperm variables defects may be observed and are termed oligoasthenozoospermia, oligoteratozoospermia, asthenoteratozoospermia or oligoasthenoteratozoospermia. In some cases, total absence of spermatozoa in the ejaculate after centrifugation may occur, a condition that is named azoospermia. It is still debatable if spermatozoa from normal, mild/moderate or severe abnormal semen may show different fertilizing abilities after ICSI [61]. Most studies report ICSI results based on the sperm source rather than on sperm defect severity. Results derived from these studies are difficult to interpret because the influence of spermatic defects is not taken into consideration. Moreover, the sperm source criteria may include spermatozoa from different etiologies. For example, ejaculated semen may either contain slightly abnormal spermatozoa from a man with a moderate varicocele or severely defective sperm from men with genetic disorders, like the Klinefelter syndrome and AZFc Y-chromosome microdeletions. Besides, spermatogenesis is very distinct in men with obstructive and non-obstructive azoospermia. While sperm production is normal in the former, it is severely abnormal, if existing, in the latter, despite the fact that in both cases ICSI may be performed using testicular spermatozoa [62]. In a recent study, our group took into consideration a different point of view by subdividing sperm deficiencies, as seen on semen analyses results, by the degree of severity, and also by the type of azoospermia [20]. We studied 313 ICSI cycles that were divided into two major groups according to the source of spermatozoa used for ICSI, i.e., ejaculated or retrieved from the epididymis/testicle. In the group of patients in which ICSI was performed with ejaculated sperm, cases were subdivided into four subgroups according to the results of the semen analysis: single defect (oligo-[O] or astheno-[A] or teratozoospermia-[T], double defect (a combination of two single defects and triple defect (OAT). In the group of azoospermic patients, cases were subdivided according to the type of azoospermia, i.e., obstructive and non-obstructive. Our results showed that significantly lower fertilization rates were obtained when either ejaculated sperm with triple defect or testicular sperm from non-obstructed azoospermic patients $(63.4 \pm 25.9 \%$ and $52.2 \pm 29.3 \%$, respectively) were used for ICSI as compared to other groups (71.3-73.6\%). There was no difference in fertilization rates when sperm from the epididymis $(74.7 \% \pm 21.2 \%)$ or the testicle $(69.1 \%$ $\pm 19.6 \%$ ) of patients with obstructive azoospermia was used for ICSI, as compared with ejaculated sperm with mild (single sperm defect; $73.2 \pm 22.1 \%$ ) to moderate (double sperm defect; $72.1 \pm 19.6 \%$ ) alterations. We also noted that embryo quality, clinical pregnancy and miscarriage rates were not statistically different between ejaculated and obstructive azoospermia (OA) groups, independent of whether the spermatozoa from obstructive azoospermic patients were obtained from the epididymis or testicle. However, embryo quality and clinical pregnancy rates were significantly lower in the group of patients with nonobstructive azoospermia when compared to the other groups (Table 5). Our results are in accordance with studies showing that severely defective sperm have a higher tendency to carry deficiencies, such as the ones related to the centrioles and genetic material, which affects the capability of the spermatozoa to activate the egg and start the formation and development of a viable embryo [63, 64]. The impaired fertilization rates after ICSI in men with non-obstructive azoospermia and oligoasthenoteratozoospermia may be explained by alterations in the early paternal effects, responsible for the initial stages of embryo development. Early paternal effects are dependent both on the spermatic cytosolic factor that dictates the completion of the oocyte meiotic division and the sperm centriole that participates in the formation of embryo mitotic fuses in early cellular

Table 5. ICSI Outcomes using Ejaculated, Epididymal and Testicular Spermatozoa (Adapted from International Braz J Urol 34, Verza Jr S and Esteves SC. Sperm defect severity rather than sperm source is associated with lower fertilization rates after intracytoplasmic sperm injection, pages 49-56, Copyright 2008, with permission)

\begin{tabular}{|c|c|c|c|c|c|c|c|}
\hline & \multicolumn{4}{|c|}{ Group 1 - Ejaculated Sperm $(n=220)$} & \multicolumn{2}{|c|}{$\begin{array}{l}\text { Group } 2 \text { - Testicular and Epididymal } \\
\text { Sperm from Azoospermic Men }(\mathrm{n}=93)\end{array}$} & \multirow{2}{*}{ p-value } \\
\hline & $\begin{array}{c}\text { Normal } \\
(n=86)\end{array}$ & $\begin{array}{l}\text { Single Defect } \\
\qquad(\mathrm{n}=\mathbf{4 1})\end{array}$ & $\begin{array}{l}\text { Double Defect } \\
\quad(n=45)\end{array}$ & $\begin{array}{l}\text { Triple Defect } \\
(n=48)\end{array}$ & OA $(n=39)$ & NOA $(n=54)$ & \\
\hline $\begin{array}{l}\text { Fertilization } \\
(\% 2 \mathrm{PN})\end{array}$ & $71.3 \pm 24.1^{\mathrm{a}}$ & $73.2 \pm 22.1^{\mathrm{b}}$ & $72.1 \pm 19.6 \mathrm{c}$ & $63.4 \pm 26.9^{\mathrm{d}}$ & $73.6 \pm 20.7^{\mathrm{e}}$ & $52.2 \pm 29.3^{\mathrm{f}}$ & $<0.05^{\text {d,f versus a, } \mathrm{a}, \mathrm{c}, \mathrm{e}}$ \\
\hline $\begin{array}{l}\text { Good embryo } \\
\text { quality* }(\%)\end{array}$ & $48.4 \pm 34.8^{\mathrm{a}}$ & $50.5 \pm 30.9^{\mathrm{b}}$ & $46.9 \pm 31.1^{\mathrm{c}}$ & $48.3 \pm 35.8^{\mathrm{d}}$ & $46.3 \pm 30.0^{\mathrm{e}}$ & $35.7 \pm 27.4^{\mathrm{f}}$ & $<0.05^{\text {f versus } \mathrm{a}, \mathrm{b}, \mathrm{c}, \mathrm{d}, \mathrm{e}}$ \\
\hline $\begin{array}{c}\text { Clinical } \\
\text { pregnancy (\%) }\end{array}$ & $40.9^{\mathrm{a}}$ & $36.6^{\mathrm{b}}$ & $44.4^{\mathrm{c}}$ & $51.0^{\mathrm{d}}$ & $51.3^{\mathrm{e}}$ & $25.9^{\mathrm{f}}$ & $0.01^{\mathrm{f} \text { versus } \mathrm{a}, \mathrm{b}, \mathrm{c}, \mathrm{d}, \mathrm{e}}$ \\
\hline Miscarriage (\%) & 14.9 & 9.1 & 12.5 & 12.0 & 20.0 & 14.3 & NS \\
\hline
\end{tabular}

One-way ANOVA was used to compare clinical and laboratorial parameters among groups. The chi-square test was used to compare pregnancy and miscarriage rates. $\mathrm{P}<0.05$ was considered significant; $\mathrm{NS}=$ not significant. $*=7-9$ blastomeres of similar size, and grades I or II cytoplasmic fragmentation on the day of embryo transfer $($ day 3 ). OA $=$ obstructive azoospermia; NOA $=$ non-obstructive azoospermia. Values are mean \pm standard deviation. 
Table 6. Mean Number of Injected Oocytes and Transferred Embryos, Live Birth Rates and Ratio of Injected Oocytes to Obtain a Live Birth after ICSI using Sperm from Men with Varying Degrees of Defective Spermatogenesis (Adapted from Fertil Steril. 88, Suppl. 1, Verza Jr S, et al. Limiting the number of oocytes to be fertilized in vitro can dramatically decrease the ability of men with severe male factor infertility to have children, page S371, Copyright 2007, with permission from Elsevier)

\begin{tabular}{|c|c|c|c|c|c|c|c|}
\hline & $\begin{array}{l}\text { Normal sperm; } \\
\quad n=168\end{array}$ & $\begin{array}{l}\text { Single defect; } \\
\quad \mathrm{n}=124\end{array}$ & $\begin{array}{l}\text { Double defect; } \\
\quad \mathrm{n}=105\end{array}$ & $\begin{array}{c}\text { Triple defect; } \\
\quad \mathrm{n}=96\end{array}$ & $\begin{array}{c}\text { OA; } \\
n=66\end{array}$ & $\begin{array}{l}\text { NOA; } \\
\mathrm{n}=67\end{array}$ & $P$-value \\
\hline Injected oocytes; n & $7.7 \pm 5.3$ & $8.7 \pm 5.5$ & $8.5 \pm 4.7$ & $8.9 \pm 6.3$ & $9.3 \pm 5.5$ & $9.9 \pm 5.7$ & NS \\
\hline Embryo transfer; $\mathrm{n}$ & $3.3 \pm 1.5$ & $3.5 \pm 1.6$ & $3.2 \pm 1.4$ & $3.2 \pm 1.6$ & $3.4 \pm 1.3$ & $3.1 \pm 1.8$ & NS \\
\hline Live birth rate; $\%$ & 32.3 & $27.9^{*}$ & $26.5^{*}$ & 31.2 & $44.0^{*}$ & $26.8^{*}$ & ${ }^{*}<0.05$ \\
\hline $\begin{array}{c}\text { No. of injected } \\
\text { oocytes/live birth }\end{array}$ & 25.6 & 33.0 & 31.0 & 29.6 & 20.5 & 38.8 & - \\
\hline \#Relative difference; \% & 34.0 & 14.9 & 20.1 & 23.7 & 47.1 & - & - \\
\hline
\end{tabular}

\# NOA versus others groups. NOA: non-obstructive azoospermia; OA: obstructive azoospermia; ejaculated sperm with single defect (oligo- [O] or astheno [A] or teratozoospermia [T]; double defect (a combination of two single defects); triple defect (a combination of the three defects [OAT]); normal (ejaculated sperm without sperm defects).

divisions [65]. Although ICSI is a formidable therapy that trespasses obstacles faced by sperm in its function as a carrier, it cannot alter the message carried by the male gamete.

Our data suggest that the reproductive potential of men undergoing assisted conception is lower in the cases of severe male factor infertility, thus highlighting the importance of the male gamete in ART. In a recent study, we aimed to compare the live birth rates after ICSI using sperm from men with varying degrees of defective spermatogenesis, and to estimate the number of oocytes that is needed to be microinjected to obtain one live birth [67]. Our data included the outcome of 626 ICSI cycles that were divided into six groups according to the source and quality of spermatozoa (Table 6). We observed that the live birth rates were significantly impaired when ICSI was performed using either testicular spermatozoa extracted from men with nonobstructed azoospermia or ejaculated sperm from men with defective spermatogenesis as compared to those with obstructive azoospermia $(\mathrm{OA})$ and normal sperm. We found that $40-50 \%$ more oocytes are to be microinjected to result in a live birth, when spermatozoa from men with defective spermatogenesis is used as compared those obtained from normal or obstructed azoospermic males. Therefore, caution should be applied when regulatory laws on assisted reproduction are undertaken, such as the ones that came to force in Italy in 2004 [66] and in Brazil in 2011. Limiting the number of oocytes to be microinjected and the ability of embryo selection and cryopreservation may be particularly harmful to men with severe male factor infertility.

\section{SURGICAL TREATMENT OF CLINICAL VARICO- CELES PRIOR TO ART}

Varicocele is the most prevalent condition affecting men with fertility-related problems, accounting for $35 \%$ of the cases [1]. Several studies have demonstrated that the surgical treatment of clinical varicoceles decreases seminal oxidative stress, increases seminal concentrations of antioxidants and improves sperm quality and the percentage of spermatozoa with intact DNA [68-70]. Recent meta-analyses on varicocelectomy also demonstrated that treatment of clinical varicoceles in infertile men with abnormal semen analyses significantly increased the chances of achieving spontaneous conception [9,71]. Even though spontaneous pregnancy remains the litmus test for evaluating varicocele treatment success, many patients with varicocele related-infertility will require ART due to the severity of sperm abnormalities and/or the presence of a significant problem affecting the female partner. Azoospermia and severe oligozoospermia in association with varicocele is reported to range from $4.3 \%$ to $13.3 \%$ [72]. The indication of varicocele repair prior to IVF/ICSI is unusual, but in certain circumstances varicocele treatment should be considered. In a recent study [73], we aimed to compare the clinical and laboratory outcomes of ICSI in a group of infertile men with treated and untreated clinical varicoceles. Our study group comprised 242 infertile men with a history of clinical varicoceles who underwent ICSI. Eighty men had a prior successful subinguinal microsurgical varicocelectomy and 162 men had any grade of clinical varicocele at the time of ICSI. Baseline semen parameters, hormone profile, testicular volume, duration of infertility, male and female partners' ages, distribution of varicocele grade and the proportion of female factor problems were not different in men with treated and untreated varicocele. Improvement in total number of motile sperm $\left(6.7 \times 10^{6}\right.$ pre, $15.4 \times 10^{6}$ post; $\left.\mathrm{p}<0.01\right)$ and reduction in sperm defect score (2.2 pre, 1.9 post; $p=0.01$ ) were observed after varicocelectomy. The normal $2 \mathrm{PN}$ fertilization ( $78 \%$ versus $66 \%$ ), clinical pregnancy $(60.0 \%$ versus $45.0 \%)$ and live birth rates $(46.2 \%$ versus $31.4 \%)$ after ICSI were significantly higher in men with treated as compared to those with untreated varicocele. Logistic regression analyses showed that the chances of achieving a clinical pregnancy (odds-ratio $=1.82 ; 95 \%$ CI: $1.06-3.15$ ) and a live birth (oddsratio $=1.87 ; 95 \%$ CI: $1.08-3.25$ ) by ICSI were significantly increased while the chance of miscarriage occurrence was reduced (odds-ratio $=0.433 ; 95 \%$ CI: $0.22-0.84$ ) if the varicocele had been treated. In the group of patients who had varicocele repair, postoperative motile sperm counts and fertilization rates by ICSI were significantly higher in men who achieved a live birth compared to those who failed to impregnate their wives (Table 7). Our data add new evidence by demonstrating that treatment of clinical varicoceles in men with a marked reduction in semen quality prior to 
Table 7. Semen Analysis Results and ICSI Outcomes in 37 vs. 43 Infertile Couples with vs. without Live Birth in whom Male Partner Underwent Varicocele Repair (Adapted from J Urol 184, Esteves SC, et al. Clinical outcomes of intracytoplasmic sperm injection in infertile men with treated and untreated clinical varicocele, pages 1442-1446, Copyright 2010, with permission from Elsevier)

\begin{tabular}{|c|c|c|c|}
\hline & $\begin{array}{l}\text { Mean; SD } \\
\text { Live Birth }\end{array}$ & $\begin{array}{l}\text { Mean; SD } \\
\text { No Live Birth }\end{array}$ & $\begin{array}{l}\text { p-value (unpaired Student's t test or } \\
\text { Pearson Chi-square test) }\end{array}$ \\
\hline Male age (years) & $35.9 ; 5.3$ & $37.4 ; 5.3$ & 0.21 \\
\hline $\begin{array}{l}\text { Male endocrine profile } \\
\qquad F S H(m I U / m L) \\
\text { Testosterone }(n g / d L)^{a} \\
\text { Testicular volume }(\text { right }+ \text { left })(\mathrm{mL})\end{array}$ & $\begin{array}{c}9.6 ; 7.7 \\
679.1 ; 793.8 \\
35.8 ; 7.7\end{array}$ & $\begin{array}{c}9.8 ; 7.6 \\
502.4 ; 799.2 \\
36.5 ; 7.8\end{array}$ & $\begin{array}{l}0.90 \\
0.32 \\
0.12\end{array}$ \\
\hline $\begin{array}{r}\text { Varicocele side }(\mathrm{n}, \%) \\
\text { Unilateral } \\
\text { Bilateral }\end{array}$ & $\begin{array}{l}17 ; 45.9 \\
20 ; 54.1\end{array}$ & $\begin{array}{l}19 ; 44.2 \\
24 ; 55.8\end{array}$ & $\begin{array}{l}0.87 \\
0.88\end{array}$ \\
\hline Volume (mL) & $2.9 ; 1.0$ & $2.7 ; 1.1$ & 0.40 \\
\hline$\%$ Strict morphology & $5.7 ; 3.0$ & $5.1 ; 2.9$ & 0.36 \\
\hline Sperm defect score & $1.8 ; 0.7$ & $1.9 ; 0.7$ & 0.52 \\
\hline $\begin{array}{l}\text { No. oocytes: } \\
\qquad \begin{array}{c}\text { Retrieved } \\
\text { Metaphase II }\end{array}\end{array}$ & $\begin{array}{l}12.4 ; 7.4 \\
10.3 ; 6.6\end{array}$ & $\begin{array}{l}12.3 ; 7.4 \\
10.2 ; 6.5\end{array}$ & $\begin{array}{l}0.95 \\
0.94\end{array}$ \\
\hline$\% 2 \mathrm{PN}$ fertilization & $79.7 ; 12.6$ & $66.0 ; 21.0$ & 0.03 \\
\hline$\%$ High-quality embryo & $47.9 ; 25.1$ & $48.2 ; 22.2$ & 0.95 \\
\hline No. embryos transferred & $3.3 ; 1.5$ & $3.2 ; 1.4$ & 0.75 \\
\hline
\end{tabular}

IVF/ICSI increases the couple's ability to conceive. The reasons for these findings seem to be related to the overall improvement in sperm quality. A significant improvement in the total number of motile sperm and a reduction in sperm defects were observed. Higher fertilization rates were also obtained after ICSI, suggesting that the improvement in pregnancy rates following varicocelectomy may be also due to functional factors not tested during standard semen analysis such as seminal oxidative stress and sperm DNA integrity.

Varicocele treatment may be also considered in men with non-obstructive azoospermia of unknown origin and concomitant clinical varicocele. We have evaluated treatment outcome after subinguinal microsurgical varicocele repair in relation to testicular histopathology in a group of non-obstructed azoospermic men with clinical varicoceles [70]. Open diagnostic testicular biopsies were performed at the same time of varicocele repair. A single piece measuring approximately $3 \times 3 \times 3 \mathrm{~mm}$ was excised and the specimens were examined by histology and classified according to the predominant pattern, as Sertoli cell-only (SCO), maturation arrest (MA) and hypospermatogenesis (HYPO). Sertoli cellonly category indicated that germinative cells were absent. Maturation arrest (MA) category was defined as absence of mature spermatozoa, despite normal early stages of sperma- togenesis. Hypospermatogenesis indicated that all stages of the spermatogenic cycle were present, including mature sperm, but there was a proportional reduction in the number of all germ cells at each level. Overall, the presence of spermatozoa in the ejaculates was achieved in $47 \%(8 / 17)$ of men after varicocele repair, but only $35 \%(6 / 17)$ had motile sperm in their ejaculates. Only men with testicular histology revealing HYPO (5/6) or maturation arrest (3/5) had improvement after surgery. Median motile sperm count for both groups were $0.9 \times 10^{6} / \mathrm{mL}$ and $0.7 \times 10^{6} / \mathrm{mL}$, respectively. The mean time for appearance of spermatozoa in the ejaculates was 5 months. One (HYPO) of 8 men who improved after surgery contributed to an unassisted pregnancy. None of the patients who had sperm in the ejaculates after varicocele repair returned to azoospermic during the mean follow-up period of 18.9 months. However, appearance of spermatozoa in the ejaculate was not achieved in any men with testicular histology diagnosis of SCO. Our findings revealed that testicular histology diagnosis was the most important predictive factor for outcome. In our series, testicular volume and preoperative serum FSH levels were not predictive of treatment outcome. Despite the induction of spermatogenesis in men with hypospermatogenesis and maturation arrest, we found that semen parameters still remained severely abnormal after the surgery, with all individuals presenting severe oligozoospermia after repeated 
routine semen analyses. In addition, $25 \%(2 / 8)$ of men who improved after surgery presented only immotile sperm in their ejaculates. Although sperm restoration is minimal and most nonobstructive azoospermic men who benefit from varicocele repair still require ICSI to conceive, it carries the possibility of ICSI to be performed using ejaculated sperm, which is technically easier and provides better results than using sperm harvested from testicular sperm extraction (TESE) [20]. Furthermore, it avoids the risk of ICSI cycle cancellation by an unsuccessful TESE or the use of donor backup.

Both studies presented in this section highlight the importance of the surgical treatment of clinical varicocele as a method to improve the couple's fertility potential by improving testicular function.

\section{REPRODUCTIVE POTENTIAL OF AZOOSPERMIC MEN IN ART}

\section{Definition and Classification}

Azoospermia is defined as an absence of spermatozoa in the ejaculate after centrifugation. This condition, which is found in $1-3 \%$ of the male population and approximately in $10 \%$ of infertile males, results in infertility but doesn't necessarily imply sterility [2]. In the case of azoospermia, two totally different clinical situations exist, i.e., obstructive and non-obstructive azoospermia. In obstructive azoospermia (OA), spermatogenesis is normal but a mechanical blockage exists in the genital tract, somewhere between the epididymis and the ejaculatory duct, or the epididymis and vas deferens are totally or partially absent. Causes of OA may be acquired or congenital. Acquired OA may be due to vasectomy, failure of vasectomy reversal, post-infectious diseases, surgical procedures in the scrotal, inguinal, pelvic or abdominal regions, and trauma. Congenital causes of $\mathrm{OA}$ include cystic fibrosis, congenital absence of the vas deferens (CAVD), ejaculatory duct or prostatic cysts and Young's syndrome [2]. Non-obstructive azoospermia (NOA) comprises a spectrum of testicular histopathology resulting from various causes that include environmental toxins, medications, genetic and congenital abnormalities, varicocele, trauma, endocrine disorders, and idiopathic. In both OA and NOA, pregnancy may be achieved through assisted reproductive techniques, i.e., in vitro fertilization associated to ICSI $[2,70]$.

\section{Sperm Retrieval Techniques for the Azoospermic Male}

Several sperm retrieval methods have been developed to collect epididymal and testicular sperm for ICSI from men with azoospermia. Either percutaneous (PESA) or microsurgical epididymal sperm aspiration (MESA) can be used to retrieve sperm from the epididymis in men with obstructive azoospermia. Testicular sperm aspiration (TESA) or extraction (TESE) can be used to retrieve sperm from the testes either in men with OA who fail epididymal retrieval. In nonobstructive azoospermia, TESA or testicular sperm extraction (TESE) using single or multiple open biopsies or microsurgical testicular sperm extraction (micro-TESE) can be used to retrieve testicular spermatozoa.

In a recent study, we evaluated 145 ICSI cycles performed in couples whose male partner had OA over a 6year period [74]. Cycles were divided according to the etiology of OA: congenital, vasectomy/failed reversal and post-infectious/trauma. Overall sperm retrieval rates (SRR) using PESA and/or TESA were 97.9\% (Table 8). Motile spermatozoa was obtained in $73.1 \%$ of the cases after the first or second PESA, and TESA was carried out after failed PESA in $29 \%$ of the cases. In our series, an adequate number of motile sperm for cryopreservation was obtained in approximately one third of the cases using percutaneous retrieval techniques. Our findings indicated that sperm retrieval is highly successful in men with OA, regardless of the cause of obstruction.

Conversely, individuals exhibiting NOA have historically been the most difficult to treat. In another study, we assessed

Table 8. Sperm Retrieval Rates and ICSI Outcomes in Obstructive Azoospermia. Retrieval Rates by Percutaneous Sperm Aspiration from the Epididymis (PESA) and Testicle (TESA) and ICSI Outcomes According to the Etiology of Obstruction (Adapted from Fertil Steril. 94, Suppl., Esteves SC, et al. Success of percutaneous sperm retrieval and intracytoplasmic sperm injection (ICSI) in obstructive azoospermic (OA) men according to the cause of obstruction, page S233, Copyright 2010, with permission from Elsevier)

\begin{tabular}{|c|c|c|c|}
\hline & Congenital $(\mathrm{N}=32)$ & Vasectomy/Failed reversal $(\mathrm{N}=59)$ & Post-infectious/Trauma $(\mathrm{N}=54)$ \\
\hline SRR using PESA; N (\%) & $21 / 32(70.0)$ & $37 / 59(62.3)$ & $44 / 54(81.5)$ \\
\hline Female Age in Years; Mean \pm SD & $31.0 \pm 5.0$ & $32.6 \pm 6.2$ & $32.9 \pm 5.9$ \\
\hline 2PN Fertilization rate; Mean (\%) & 64.1 & 65.3 & 59.3 \\
\hline Number of Embryos Transferred; Mean & 2.9 & 2.6 & 3.0 \\
\hline Clinical Pregnancy Rate per Transfer; N (\%) & $16 / 29(55.2)$ & $26 / 59(44.0)$ & $23 / 53(43.4)$ \\
\hline Miscarriage Rate; N (\%) & $5 / 16(31.2)$ & $7 / 26(26.7)$ & $3 / 23(13.2)$ \\
\hline Live Birth Rate per Transfer; N (\%) & $11 / 29(37.8)$ & $19 / 59(32.2)$ & $20 / 53(37.7)$ \\
\hline
\end{tabular}

SRR: sperm retrieval rate; One-way ANOVA and Chi-square test used to compare SRR and ICSI outcomes among groups. P-values were not statistically significant for all variables analyzed. 
the success rates of sperm retrieval in a group of 176 men with NOA [75]. Retrievals were performed by TESA or micro-TESE and testicular histopathology results were available for 131 individuals. SRR were analyzed according to the histology patterns, i.e., hypospermatogenesis (hypo), maturation arrest (MA) and Sertoli cell-only (SCO), and to the etiology of NOA. Overall SRR rates by TESA and micro-TESE were similar (55.7\%). However, SRR by TESA was significantly lower in cases of maturation arrest (MA) and Sertoli-cell only (SCO) combined (22.8\%) compared to micro-TESE (39.2\%). Both methods yielded similar SRR $(100 \%)$ in cases of testicular histology showing hypospermatogenesis. Successful sperm retrieval rates were in the range of $50-70 \%$ in most etiology-specific causes of NOA. Testicular spermatozoa were obtained in all etiology-specific causes of NOA, such as varicocele, cryptorchidism, orchitis, genetic, endocrine and gonadotoxic-induced, and results were not different in etiology-specific subgroups of NOA (Table 9). According to our data, micro-TESE performed

Table 9. Sperm Retrieval Rates in Non-Obstructive Azoospermia. Percutaneous Testicular Sperm Aspiration (TESA) and Microsurgical Testicular Sperm Extraction (micro-TESE) According to the Testicular Histopathology Results and the Etiology of Azoospermia (Adapted from Fertil Steril. 94, Suppl., Esteves SC, et al. Sperm retrieval rates (SRR) in nonobstructive azoospermia (NOA) are related to testicular histopathology results but not to the etiology of azoospermia, page S132, Copyright 2010, with permission from Elsevier)

\begin{tabular}{|c|c|}
\hline & $\begin{array}{l}\text { Presence of Testicular } \\
\text { Spermatozoa; N }(\%)\end{array}$ \\
\hline \multicolumn{2}{|l|}{ By Method } \\
\hline TESA ( $\mathrm{N}=61)$; overall SRR, $\mathrm{N}(\%)$ & $34 / 61(55.7)$ \\
\hline Нуро & $26 / 26(100.0)$ \\
\hline MA & $2 / 6(33.3)$ \\
\hline $\mathrm{SCO}$ & $6 / 29(20.7)$ \\
\hline $\begin{array}{c}\text { Micro-TESE }(\mathrm{N}=70) \\
\text { overall SRR, } \mathrm{N}(\%)\end{array}$ & $39 / 70(55.7)$ \\
\hline Нуро & $19 / 19(100.0)$ \\
\hline MA & $7 / 12(58.3)$ \\
\hline $\mathrm{SCO}$ & $13 / 39(33.3)$ \\
\hline \multicolumn{2}{|l|}{ By Cause of $\mathrm{NOA}$} \\
\hline Varicocele $(\mathrm{N}=66)$ & $45 / 66(68.2)$ \\
\hline Genetic $(\mathrm{N}=12)$ & $6 / 12(50.0)$ \\
\hline Cryptorchidism $(\mathrm{N}=19)$ & $12 / 19(63.1)$ \\
\hline Idiopathic $(\mathrm{N}=63)$ & $33 / 63(52.4)$ \\
\hline Radio/chemotherapy $(\mathrm{N}=6)$ & $3 / 6(50)$ \\
\hline Orchitis/Gonadotoxin/Endocrine $(\mathrm{N}=10)$ & $10 / 10(100.0)$ \\
\hline Overall SRR; N (\%) & $109 / 176(61.9)$ \\
\hline
\end{tabular}

better than TESA in MA and SCO histology subgroups, where focus of spermatogenesis were identified using the operating microscope. Testicular spermatozoa were obtained in all etiology-specific causes of NOA, and SRR seemed to be independent of its cause. Testicular histology results, if available, are useful to predict the chances to retrieve sperm in men with NOA. However, sperm can be obtained even in the worst scenario of Sertoli-cell only.

In our experience involving 314 individuals with obstructive and non-obstructive azoospermia, the overall sperm retrieval rates (SRR), defined as successful surgical collection of spermatozoa for ICSI, were significantly higher in the group of OA $(\mathrm{SRR}=97.9 \% ; \mathrm{N}=142 / 145)$ compared to NOA (SRR $=55.2 \% ; \mathrm{N}=95 / 172)$ [76]. Logistic regression analysis revealed that the chances of retrieving spermatozoa were markedly increased in couples whose male partner had obstructive rather than non-obstructive azoospermia (odds ratio $=43.0 ; 95 \%$ confidence interval 10.3-179.5). Our data clearly indicate that sperm retrieval rates are dependent on the azoospermia category, i.e., obstructive or non-obstructive.

\section{Clinical Outcome of ICSI in the Azoospermic Male}

In recent studies, we evaluated the reproductive outcomes by ICSI using surgically-retrieved sperm in men with OA and NOA [20,74-76]. PESA/TESA or TESA/microTESE was used in the attempt to retrieve spermatozoa in OA and in NOA cases, respectively. ICSI was performed using surgically-retrieved epididymal or testicular spermatozoa, as appropriate. Out of 2,136 couples undergoing ICSI from 2002 and 2008, $145(6.8 \%)$ and $172(8.0 \%)$ male partners had obstructive and non-obstructive azoospermia, respectively. We found that ICSI outcomes in men with OA were independent of the cause of obstruction [74]. Similar fertilization rates after ICSI using epididymal/testicular spermatozoa and embryo formation were obtained in different etiology categories of vasectomy/failed reversal, congenital or post-infectious/trauma (Table 8). Moreover, clinical pregnancy, miscarriage and live birth rates were comparable, regardless of the cause of obstruction. In another study, we examined the reproductive potential of epididymal and testicular spermatozoa retrieved from men with OA. Laboratory and clinical ICSI outcomes did not differ between microinjections performed using either epididymal or testicular sperm (Table 10) [20]. The clinical outcomes of ICSI using testicular or epididymal sperm aspirated from men with OA were similar than those obtained with ejaculated sperm from normal men and those presenting with mild/moderate semen abnormalities (Table 5).

Comparison of ART outcome between men with obstructive and non-obstructive azoospermia, on the other hand, yelled different results. From our data involving 314 ICSI cycles, fertilization rates were significantly higher in the group of men with OA $(60.5 \%)$ compared to NOA $(51.1 \%$; $\mathrm{P}<0.01)$ [76]. The overall pregnancy rates, defined as the live birth rate (LBR) per transfer, were $38.2 \%(50 / 131)$ and $25.0 \%(22 / 88)$ in the groups of men with OA and NOA, respectively $(\mathrm{P}=0.03)$. The chances of achieving a live birth by ICSI (odds ratio $=1.86$; $95 \%$ confidence interval 1.03 2.89) were significantly increased in couples whose male partner had obstructive rather than non-obstructive azoospermia. 
Table 10. ICSI Outcomes Using Spermatozoa Retrieved from the Epididymis or the Testicle of Men with Obstructive (AO) (Adapted from International Braz J Urol 34, Verza Jr S and Esteves SC. Sperm defect severity rather than sperm source is associated with lower fertilization rates after intracytoplasmic sperm injection, pages 49-56, Copyright 2008, with permission)

\begin{tabular}{|c|c|c|}
\hline Source of sperm for ICSI & \multicolumn{2}{|c|}{ Obstructive Azoospermia } \\
\hline Number of Cycles; $N=107$ & 93 & 14 \\
\hline Female Age in Years; Mean \pm SD & $32.6 \pm 5.3$ & $32.1 \pm 5.4$ \\
\hline 2PN Fertilization Rate; \% & $66.0^{\mathrm{a}}$ & $56.6^{\mathrm{a}}$ \\
\hline Cleavage Rate; $\%$ & 99.4 & 95.7 \\
\hline Number of Embryos Transferred; Mean & 2.8 & 3.3 \\
\hline Clinical Pregnancy Rate per Transfer; N (\%) & $45 / 88(51.1)$ & $7 / 14(50.0)$ \\
\hline Miscarriage Rate; N (\%) & $11 / 45(24.4)$ & $1 / 7(14.3)$ \\
\hline Live Birth Rate per Transfer; N (\%) & $34 / 88(38.6)$ & $6 / 14(42.8)$ \\
\hline
\end{tabular}

Values expressed as means for fertilization, cleavage and embryo quality rates.

* 7-9 blastomeres of similar size, and grades I or II cytoplasm fragmentation on the day of embryo transfer (day 3).

One-way ANOVA and Chi-square test used to compare laboratory and clinical ICSI parameters between groups. Statistically significant results were not obtained for any of the outcome measures. $\mathrm{P}<0.05$ was considered significant.

As previously discussed, the laboratory and clinical outcomes of ICSI using testicular sperm extracted from men with NOA were significantly lower than all other categories including ejaculated specimens obtained from men with varying degrees of defective spermatogenesis and epididymal/testicular sperm from men with OA (Table 5) [20]. From these data, it is suggested that testicular spermatozoa of men with severely impaired spermatogenesis have decreased fertility potential, and may have a higher tendency to carry deficiencies such as the ones related to the centrioles and genetic material, which ultimately affect the capability of the male gamete to activate the egg and trigger the formation and development of a normal zygote and a viable embryo [20].

\section{EXPERT COMMENTARY}

The aim of this article is to present our 10-year experience with ART in the management of severe male factor infertility. Currently, ART is the only option for most men with severe male factor infertility to have biological offspring. Success has been achieved with this treatment modality in several male infertility conditions, including immunological infertility, severe oligozoospermia/other sperm deficiencies and azoospermia. It should be noted, however, that the reproductive potential of infertile men in ART varies according to severity of infertility. Therefore, strategies to optimize ART outcomes are encouraged. Many men with severe male factor infertility have lifestyle choices that include smoking, obesity, poor diet intake, use of gonadotoxic medication, and exposure to environmental toxins. These conditions are often associated with increased systemic or seminal oxidative stress and may have a negative synergistic effect on ART outcomes. We strongly advise patients enrolled in our ART program to improve their reproductive health by lifestyle modifications. Treatment of clinical varicocele prior to ART is another example of the impact of an intervention aiming to improve the overall male reproductive health.
When dealing with azoospermic males, the adoption of strict criteria to diagnose obstructive azoospermia is crucial for obtaining high success retrieval rate in the range of 90$100 \%$ using percutaneous techniques. Most cases of PESA failures are not necessarily technical failures since immotile spermatozoa can be retrieved. However, PESA may fail in certain cases of epididymal fibrosis due to multiple PESA attempts or post-infection. In these cases, TESA or PESA at the contralateral epididymis can be applied successfully. We routinely perform sperm retrieval under local anesthesia, with or without intravenous sedation. Percutaneous sperm retrieval techniques can be performed both for diagnostic and for therapeutic purposes. In the latter, sperm retrieval is often carried out at the same day of oocyte retrieval or at the day before. Patients are discharged one hour later and can return to normal activities in the same day. Oral analgesics are prescribed but pain complaint is minimal. The most common complication is fibrosis at the aspiration site. Some authors advocate that microsurgical epididymal sperm aspiration (MESA) allows the collection of larger and cleaner quantities of sperm than PESA, but this debate seems trivial. In our series, cumulative successful retrieval rate after PESA and/or TESA was higher than $95 \%$, and an adequate number of motile sperm for cryopreservation was obtained in approximately one third of the cases. Although cryopreservation rate after PESA is not high, repeated aspirations can be carried out in men with OA with minimal morbidity and lower cost compared to MESA. When gradient centrifugation is chosen for PESA sperm processing, we spare part of the sample to be processed by simple washing. The reason is the unpredictability of gradient centrifugation to recover motile sperm in such cases. If recovery is less than desired, we can rely on the washed sample to select motile sperm for ICSI. Due to the relatively low sperm yields in PESA and TESA, it is important to use low volumes of media during sperm processing and wash the sample only once. Centrifugation force and time should be carefully controlled to avoid jeopardizing the often compromised sperm motility. 
Although not absolute, we consider testicular histology as the best predictor for successful sperm retrieval in NOA men. The probability of obtaining sperm varies according to the testicular histopathology results. In NOA, our choice is to use micro-TESE rather than conventional TESE or TESA in cases of maturation arrest or SCO. Micro-TESE is also the safest technique regarding post-operative complications. Proper identification of testicular vessels under the tunica albuginea is made prior to the placement of an incision into the testis. The use of optical magnification and microsurgery technique allows the preservation of intratesticular blood supply, as well as the identification of tubules more likely to harbor sperm production. Therefore, efficacy of sperm retrieval is improved while the risks of large tissue removal are minimized. Nonetheless, our experience with TESA in NOA indicates that it may be highly successful in men with diagnostic testicular biopsy histopathology showing hypospermatogenesis or in those with a previous successful TESA attempt. However, if TESA fails, we neither perform a second aspiration in the same testis, at the same operative time, nor convert it to an open procedure, to avoid the risk of hematoma and testicular injury. From our clinical experience, it is very difficult to identify enlarged seminiferous tubules in such cases, even using the operating microscope, because extensive bleeding is often seen. In these occasions, we opt to perform TESA or TESE at the contralateral testis. For NOA patients without previous diagnostic testicular biopsy or TESA attempt, our choice is to perform sperm extraction using micro-TESE. Tissue removal in microTESE is often minimal as compared to conventional TESE; the small amount of tissue extracted facilitates sperm processing in the IVF lab. Selection of spermatozoa from a smaller population of contaminating testicular cells allows more ease and greater speed for sperm pick-up and injection process, as well as alleviates contamination and blockage of the injection needle with cells and debris. It is far less technically demanding and labor-intensive to extract spermatozoa from small volume specimens than large pieces of testicular tissue that must be dissected, red-blood cells lysed, and the rare spermatozoa searched for in a tedious fashion under an inverted microscope. TESE sperm processing may be incredibly labor-intensive and the searching process may miss the rare spermatozoa within a sea of seminiferous tubules and other cells. TESE/micro-TESE may be scheduled either for the day of oocyte collection and ICSI or the day before. In the latter, processed specimens are incubated in a closed HEPES-buffered culture system (microdrops under mineral oil) at room temperature, inside a laminar flow cabinet or in a cleanroom for a maximum of 48 hours, to avoid bacterial contamination. Culture of specimens at $37^{\circ} \mathrm{C}$ inside the incubator should be avoided since contamination with scrotum skin derived-bacteria is often seen. From our data, optimal fertilization by ICSI using surgically-retrieved sperm is obtained when the time frame from hCG administration to microinjection does not exceed 43h. Testicular tissue sperm processing, searching and selection of viable spermatozoa for ICSI may take several hours in NOA cases. Our laboratory takes approximately 11.6 minutes to handle a single testicular spermatozoon from processing to microinjection in NOA, but only 5.5 minutes in OA. In other words, the average time required to perform ICSI in a standard NOA treatment cycle involving 8-12
metaphase-II oocytes is approximately 2 hours. Therefore, we elect to perform micro-TESE the day before oocyte collection when a busy next day IVF laboratory workload is anticipated. Additionally, we recommend that two laboratory technicians work together during the initial processing steps (one mincing the tubules and the other searching for spermatozoa) to speed up the searching process and to allow a faster feedback to the surgeon who may decide to terminate the procedure if sperm is found or to continue dissecting the seminiferous tubules. Our laboratory performs the processing of testicular specimens by mincing and shredding the whole tissue instead of using enzymatic digestion. The mechanical preparation has the advantage of being fast, requiring about 15-30 minutes, while enzymatic digestion is more time consuming, requiring at least 4 hours for sperm preparation.

\section{FIVE-YEAR REVIEW}

Development in ART has been remarkable in the past few years. Over half a million ART treatment cycles are performed annually worldwide and the infertile male is a significant source for this increasing demand [77-79]. Several medical conditions affecting the male fertility status are now treated by ART, especially by the combination of in vitro fertilization and intracytoplasmic sperm injection. Nonetheless, the reproductive potential of sperm from men with defective spermatogenesis is decreased [20], while it is suggested that there is an increased risk of transmitting genetic and epigenetic defect for the embryo [80, 81]. Factors associated with higher numbers of aneuploid sperm are cigarette smoking, elevated follicle-stimulating hormone level, severe oligozoospermia and teratozoospermia [81]. Therefore, efforts should be made to improve the male health status prior to embarking on ART because current evidence suggests that fertility optimization may improve treatment outcomes $[73,82]$. Men with varicocele related-infertility who require ART due to the severity of sperm abnormalities may benefit of varicocele repair prior to IVF/ICSI [73]. Even men with non-obstructive azoospermia with favorable testicular histopathology may restore sperm to the ejaculate after repair of clinical varicoceles [70, 83]. Sperm restoration, although minimal, yields the possibility of IVF/ICSI without the need of sperm retrieval techniques. In the group of patients who are still azoospermic after varicocelectomy, the chances of sperm extraction may be increased, and as a result the couple's chance for pregnancy, if the varicocele had been treated before sperm retrieval and assisted conception [82].

The laboratory management of male infertility cases involving very poor quality semen specimens requires special attention. After collecting ejaculates or epididymal fluid or testicular tissue, laboratory processing techniques are used to remove contaminants, cellular debris, noxious microorganisms and red blood cells. Processed spermatozoa may be either used for ICSI or cryopreserved. Spermatozoa collected from men with severely impaired spermatogenesis are often compromised and fragile. Therefore, adherence to state of the art laboratory techniques and quality control are recommended to avoid jeopardizing the sperm fertilizing potential in treatment cycles [21, 25, 28, 32]. 
Spermatozoa can be retrieved either from the epididymis or the testis, depending on the type of azoospermia, using different surgical methods such as PESA, TESA, TESE and micro-TESE. Meta-analysis results demonstrated no significant difference in any outcome measure between the use of epididymal or testicular sperm in men with obstructive azoospermia [84]. The etiology of the obstruction and the use of fresh or frozen-thawed epididymal/testicular sperm do not seem to affect ICSI outcomes in terms of fertilization, pregnancy, or miscarriage rates [74]. Sperm can be easily retrieved in cases of OA regardless of the surgical method while the best sperm retrieval technique in non-obstructive azoospermia is yet to be established. Current recommendations are based on cumulative evidence provided by descriptive, observational and controlled studies. Efficiency of TESA for retrieving spermatozoa in NOA varies from 10$30 \%$ [85], except in the favorable cases of men with previous successful TESA or testicular histopathology showing hypospermatogenesis. In such individuals, sperm retrieval rates by TESA are in the range of $70-100 \%$ [86-88]. In a recent systematic review the mean reported SRR for TESE was $49.5 \%$. TESE with multiple biopsies resulted in higher SRR than fine-needle aspiration, a variation of TESA, especially in cases of Sertoli-cell-only (SCO) syndrome and maturation arrest [89]. In NOA, current evidence suggests that micro-TESE performs better than conventional TESE or TESA in cases of SCO, where tubules containing active focus of spermatogenesis can be identified [75]. Tissue removal in micro-TESE is often 50 to 70 -fold less than conventional TESE [89, 90-92], and the small amount of tissue extracted facilitates sperm processing. Micro-TESE also appears to be the safest technique regarding postoperative complications [92-94]. The clinical outcomes of ICSI using testicular sperm extracted by TESA or microTESE in NOA are significantly lower than those obtained with either ejaculated or epididymal/testicular sperm from men with OA [20]. From the limited data available, it is suggested that the sperm retrieval technique itself has no impact on ICSI success rates [89]. However, frozen-thawed surgically-retrieved sperm from NOA men have significantly impaired reproductive potential than fresh ones $[65,84]$. Meta-analysis results showed that fertilization rates by ICSI remained similar, but implantation was significantly higher (by $73 \%$ ) with the use of fresh compared to frozen-thawed testicular sperm [84]. The concern that ICSI using sperm retrieved from men with either OA or NOA might be associated with increased risk for birth defects is still unresolved. In general, in vitro fertilization is associated with an increased risk of multiple pregnancies and congenital abnormalities (including hypospadias) [95]. ICSI in particular carries an increased risk of endocrine abnormalities, as well as epigenetic imprinting effects [95]. Although the absolute risk of any of these conditions remains low [95-98], current data is limited and study populations are heterogenic. It is therefore recommended that well-defined groups of ICSI with ejaculated sperm, ICSI with epididymal sperm and ICSI with testicular sperm, and a control group of naturally conceived children are closely followed up.

Despite the reduced ART success rates obtained with sperm from men with severely impaired spermatogenesis, some countries are either considering or have already implemented regulatory laws to restrict the production of embryos in vitro by limiting the number of oocytes to be fertilized. Recent studies critically evaluated the impact of these restrictions on ART outcome in couples affected by severe male factor infertility [66, 67, 99]. They concluded that limiting the number of treated oocytes to three per ICSI cycle significantly reduces the chance of transferring good quality embryos and thus achieving a live birth in cases of severe male factor infertility. Cryptozoospermic and NOA men are particularly affected by these restrictions.

\section{KEY POINTS}

- Assisted Reproductive Technology (ART) is a formidable treatment modality for most untreatable causes of male infertility, including immunologic infertility, severe oligozoospermia and azoospermia.

- $\quad$ Attention to some technical aspects during intracytoplasmic sperm injection (ICSI) may optimize treatment outcome in cases of severe male factor infertility: (i) men with severe oligozoospermia/ cryptozoospermia men should consider sperm cryobanking to avoid the risk of collecting very poor semen specimens at the time of the ICSI; (ii) the optimal time interval for microinjection is $38-42$ hours after $\mathrm{hCG}$ administration; (iii) selection of sperm with adequate morphology for ICSI can be accomplished by using $400 \mathrm{X}$ optical magnification; it is also possible to identify and to perform microinjections at the oocyte vegetal pole to minimize the risk of damaging the oocyte meiotic spindle; (iv) air quality control in the IVF laboratory and critical areas may positively impact on embryo development and pregnancy results.

- Antisperm antibodies are not detrimental to ICSI outcome.

- The reproductive potential of infertile males undergoing ART is related to the severity of sperm abnormalities. Live birth rates are lower when ICSI is performed using either testicular spermatozoa extracted from men with non-obstructed azoospermia or ejaculated sperm from men with defective spermatogenesis as compared to those with obstructive azoospermia (OA) and normal sperm.

- $\quad$ Surgical repair of clinical varicocele should be considered in men with severely abnormal spermatogenesis or non-obstructed azoospermia prior to embarking on ART. Varicocelectomy may increase the chance of live birth by ICSI and restore spermatogenesis in nonobstructive azoospermic men (NOA) with testicular histopathology exhibiting maturation arrest or hypospermatogenesis.

- Sperm retrieval is simple and highly successful in men with OA, regardless of the cause of obstruction. In NOA, testicular spermatozoa can be obtained in all etiology-specific causes; efficiency rates are approximately $50 \%$ and do not differ according to the etiology-specific subgroups. Testicular histology results are predictive of sperm retrieval in NOA; micro-TESE performs better than TESA in maturation arrest and Sertoli cell-only histology subgroups. Sperm retrieval 
rates are significantly higher in men with obstructive compared to non-obstructive azoospermia.

- The clinical outcome of ART in azoospermic men is dependent on the azoospermia being obstructive or non-obstructive. In OA, ICSI results are independent of the cause of obstruction. ICSI using testicular or epididymal sperm aspirated from men with OA are comparable. Conversely, the chances of achieving a live birth by ICSI are significantly reduced in couples whose male partner have non-obstructive azoospermia.

\section{ACKNOWLEDGEMENTS}

None Declared.

\section{CONFLICT OF INTEREST}

None Declared.

\section{REFERENCES}

[1] Vital and Health Statistics, series 23, no.26, CDC. Available from: http://www.cdc.gov. [cited: $10^{\text {th }}$ Dec 2009].

[2] Esteves SC. Infertilidade masculina. Rhoden EL, Eds. Urologia no consultório. Porto Alegre: Artmed Editora $1^{\text {st }}$ ed. 2009; pp. 470500 .

[3] Shefi S, Turek PJ. Definition and current evaluation of subfertile men. Int Braz J Urol 2006; 32 (4): 385-97.

[4] Vine MF. Smoking and male reproduction: A review. Int J Androl 1996; 19(6): 323-37.

[5] Auger J, Eustache F, Andersen AG, et al. Sperm morphological defects related to environment, lifestyle and medical history of 1001 male partners of pregnant women from four European cities. Hum Reprod 2001; 16(12): 2710-7.

[6] Kenkel S, Rolf C, Nieschlag E. Occupational risks for male fertility: An analysis of patients attending a tertiary referral centre. Int J Androl 2001; 24(6): 318-26.

[7] Kefer JC, Agarwal A, Sabanegh E. Role of antioxidants in the treatment of male infertility. Int J Urol 2009; 16(5): 449-57.

[8] Cabler S, Agarwal A, Flint M, Du Plessis SS. Obesity: modern man's fertility nemesis. Asian J Androl 2010; 12(4): 480-9.

[9] Marmar JL, Agarwal A, Prabakaran S, et al. Reassessing the value of varicocelectomy as a treatment for male subfertility with a new meta-analysis. Fertil Steril 2007; 88(3): 639-48.

[10] Esteves SC, Schneider DT, Verza Jr S. Influence of antisperm antibodies in the semen on intracytoplasmic sperm injection outcome. Int Braz J Urol 2007; 33(6): 795-802.

[11] Sharma RK, Sabanegh E, Mahfouz R, Gupta S, Thiyagarajan A, Agarwal A. TUNEL as a test for sperm DNA damage in the evaluation of male infertility. Urology 2010; 76(6): 1380-6.

[12] Bates CA. Antisperm antibodies and male subfertility. Br J Urol 1997; 80(5): 691-7.

[13] Mahfouz R, Sharma R, Thiyagarajan A, et al. Semen characteristics and sperm DNA fragmentation in infertile men with low and high levels of seminal reactive oxygen species. Fertil Steril 2010; 94(6): 2141-6.

[14] Zegers-Hochschild F, Nygren KG, Adamson GD, et al. The ICMART glossary on ART terminology. Hum Reprod 2006; 21(8): 1968-70.

[15] Palermo G, Joris H, Devroey P, Van Steirteghem AC. Pregnancies after intracytoplasmic sperm injection of single spermatozoan into an oocyte. Lancet 1992; 340 (8810): 17-8.

[16] Silber S, Nagy ZP, Liu J, Godoy H, Devroey P, Van Steirteghem AC. Conventional in-vitro fertilization versus intracytoplasmic sperm injection for patients requiring microsurgical sperm aspiration. Hum Reprod 1994; 9(9): 1705-9.
[17] Devroey P, Liu J, Nagy ZP, et al. Pregnancies after testicular extraction (TESE) and intracytoplasmic sperm injection (ICSI) in non-obstructive azoospermia. Hum Reprod 1995; 10(6): 1457-60.

[18] Esteves SC, Schertz JC, Verza Jr S, Schneider DT, Zabaglia SFC. A comparison of menotropin, highly-purified menotropin and follitropin alfa in cycles of intracytoplasmic sperm injection. Reprod Biol Endocrinol 2009; 7:111.

[19] Rhoden E, Soares J, Esteves S. O que o laboratório pode fazer pelo espermatozóide. II Consenso Brasileiro de Infertilidade Masculina. Int Braz J Urol 2003; 29 (Suppl 5): 50-5.

[20] Verza Jr S, Esteves SC. Sperm defect severity rather than sperm source is associated with lower fertilization rates after intracytoplasmic sperm injection. Int Braz J Urol 2008; 34(1): 4956.

[21] Esteves SC, Guidi AR, Zabaglia SFC, Verza Jr S. Intracytoplasmic sperm injection: optical magnification during sperm selection and microinjection affects fertilization, cleavage, and pregnancy rates [abstract]. Fertil Steril 2003; 80 (Suppl 3): 124.

[22] Schneider DT, Verza Jr S, Esteves SC. Single or sequential medium are equally effective for the culture of human embryos to the blastocyst stage: a pilot study [abstract]. Fertil Steril 2009; 92 (3 Suppl): 231-2.

[23] Veeck LL. The morphological assessment of human oocytes and early concept. In: Keel B, Webster BW, Eds. Laboratory Diagnosis and Treatment of Infertility. Boca Raton: CRC Press 1990; pp. 35369.

[24] Esteves SC, Verza Jr S, Gomes AP. Control of air pollution in assisted reproductive technology laboratory and adjacent areas improves embryo formation, cleavage and pregnancy rates and decreases abortion rate: Comparison between a class 100 (ISO 5) and a class 1000 (ISO 6) cleanroom for micromanipulation and embryo culture [abstract]. Fertil Steril 2004; 82 (Suppl 2): 259-60.

[25] Esteves SC, Verza Jr S, Gomes AP. Comparison between International Standard Organization (ISO) type 5 and type 6 cleanrooms combined with volatile organic compounds filtration system for micromanipulation and embryo culture in severe male factor infertility [abstract]. Fertil Steril 2006; 86 (3 Suppl): 353-4.

[26] Hsu YL, Kuo BJ. Evaluations of emotional reactions and coping behaviors as well. J Nurs Res 2002; 10 (4): 291-302.

[27] Peterson BD, Newton CR, Feingold T. Anxiety and sexual stress in men and women undergoing infertility treatment. Fertil Steril 2007; 88 (4): 911-4.

[28] Feijo C, Nascimento J, Verza Jr S, Esteves SC. Difference of semen parameters between sperm samples collected for instant use in ICSI cycles compared to previous sperm analysis in the same men [abstract]. Fertil Steril 2008; 90 (Suppl): 316.

[29] Dozortsev D, Nagy P, Abdelmassih S, et al. The optimal time for intracytoplasmic sperm injection in the human is from 37 to 41 hours after administration of human chorionic gonadotropin. Fertil Steril 2004; 82(6): 1492-6.

[30] Yuzpe AA, Liu Z, Fluker MR. Rescue intracytoplasmic sperm injection (ICSI) - salvaging in vitro fertilization (IVF) cycles after total or near total fertilization failure. Fertil Steril 2000; 73(6): 1115-9.

[31] Tucker MJ, Morton PC, Wright G, Ingargiola PE, Jones AE, Sweitzer CL. Factors affecting success with intracytoplasmic sperm injection. Reprod Fertil Dev 1995; 7(2): 229-36.

[32] Schneider DT, Prado Gomes A, Verza Jr S, Esteves SC. Optimal time interval for intracytoplasmic sperm injection after human chorionic gonadotropin administration in severe male factor infertility [abstract]. Fertil Steril 2006; 86 (3 Suppl): 155.

[33] Berkovitz A, Eltes F, Lederman H, et al. How to improve IVF-ICSI outcome by sperm selection. Reprod Biomed Online 2006; 12(5): 634-8.

[34] Awata S, Himeno N, Takemoto Y, et al. No increased incidence of chromosomal aberrations in human spermatozoa with large vacuoles by injection into mouse oocytes. J Mammal Ova Res 2008; 25(4): 240-5.

[35] Boone WR, Johnson JE, Locke AJ, Crane MM IV, Price TM. Control of air quality in an assisted reproductive technology laboratory. Fertil Steril 1999; 71(1): 150-4.

[36] Wang H, Wang R, Liu J. Decreased in vitro fertilization and cleavage rates after an equipment error during $\mathrm{CO}_{2}$ calibration. Fertil Steril 2000; 73: 1247-9. 
[37] Cohen J, Gilligan A, Esposito W, Schimmel T, Dale B. Ambient air and its potential effects on conception in vitro. Hum Reprod 1997; 12(8): 1742-9.

[38] Higdon HL, Blackhurst DW, Boone WR. Incubator management in an assisted reproductive technology laboratory. Fertil Steril 2008; 89(3): 703-10.

[39] Turek PJ. Immunopathology and infertility. In: Lipshultz LI, Howards SS, Eds. Infertility in the male. St. Louis: Mosby-Year Book Inc 1997; pp. 305-25.

[40] Srivastava PN, Sheikhnejad RG, Fayrer-Hosken R, Malter H, Brackett BG. Inhibition of fertilization of the rabbit ova in vitro by the antibody to the inner acrosomal membrane of rabbit spermatozoa. J Exp Zool 1986; 238(1): 99-102.

[41] Shibahara H, Burkman LJ, Isojima S, Alexander NJ. Effects of sperm-immobilizing antibodies on spermzona pellucida tight binding. Fertil Steril 1993; 60(3): 533-9.

[42] Rajah SV, Parslow JM, Howell RJ, Hendry WF. The effects on invitro fertilization of autoantibodies to spermatozoa in subfertile men. Hum Reprod 1993; 8(7): 1079-82.

[43] Sukcharoen N, Keith J. The effect of the antisperm auto-antibodybound sperm on in vitro fertilization outcome. Andrologia 1995; 27(5): 281-9.

[44] Chang TH, Jih MH, Wu TC. Relationship of sperm antibodies in women and men to human in vitro fertilization, cleavage, and pregnancy rate. Am J Reprod Immunol 1993; 30 (2-3): 108-12.

[45] Ford WC, Williams KM, McLaughlin EA, Harrison S, Ray B, Hull MG. The indirect immunobead test for seminal antisperm antibodies and fertilization rates at in-vitro fertilization. Hum Reprod 1996; 11(7): 1418-22.

[46] Clarke GN. Induction of the shaking phenomenon by IgA class antispermatozoal antibodies from serum. Am J Reprod Immunol Microbiol 1985; 9(1): 12-4.

[47] Lahteenmaki A, Veilahti J, Hovatta O. Intra-uterine insemination versus cyclic, low-dose prednisolone in couples with male antisperm antibodies. Hum Reprod 1995; 10(1): 142-7.

[48] Shulman S. Therapy of immunological infertility. J Immunol Immunopharmacol 1986; 6: 95.

[49] Hendry WF, Treehuba K, Hughes L, et al. Cyclic prednisolone therapy for male infertility associated with autoantibodies to spermatozoa. Fertil Steril 1986; 45(2): 249-54.

[50] Agarwal A. Treatment of immunological infertility by sperm washing and intrauterine insemination. Arch Androl 1992; 29(3): 207-13.

[51] Yeh WR, Acosta AA, Seltman HJ, Doncel G. Impact of immunoglobulin isotype and sperm surface location of antisperm antibodies on fertilization in vitro in the human. Fertil Steril 1995; 63(6): 1287-92.

[52] Acosta AA, van der Merwe JP, Doncel G, et al. Fertilization efficiency of morphologically abnormal spermatozoa in assisted reproduction is further impaired by antisperm antibodies on the male partner's sperm. Fertil Steril 1994; 62(4): 826-33.

[53] Vazquez-Levin MH, Notrica JA, Polak de Fried E. Male immunologic infertility: sperm performance on in vitro fertilization. Fertil Steril 1997; 68(4): 675-81.

[54] de Almeida M, Gazagne I, Jeulin C, et al. In-vitro processing of sperm with autoantibodies and in-vitro fertilization results. Hum Reprod 1989; 4(1): 49-53.

[55] Lenzi A, Gandini L, Claroni F, Lombardo F, Morrone S, Dondero F: Immunological usefulness of semen manipulation for artificial insemination homologous (AIH) in subjects with antisperm antibodies bound to sperm surface. Andrologia 1988; 20(4): 31421.

[56] Schneider DT, Feijó CM, Verza Jr S, Esteves SC. Títulos de anticorpos antiespermatozóides antes e após o processamento seminal pela técnica do gradiente descontínuo coloidal [abstract]. Int Braz J Urol 2005; 30 (Suppl 1): 87.

[57] Nagy ZP, Verheyen G, Liu J, et al. Results of 55 intracytoplasmic sperm injection cycles in the treatment of male-immunological infertility. Hum Reprod 1995; 10(7): 1775-80.

[58] Lahteenmaki A, Reima I, Hovatta O. Treatment of severe male immunological infertility by intracytoplasmic sperm injection. Hum Reprod 1995; 10(11): 2824-8.

[59] Clarke GN, Bourne H, Baker HW. Intracytoplasmic sperm injection for treating infertility associated with sperm autoimmunity. Fertil Steril 1997; 68(1): 112-7.
[60] Check ML, Check JH, Katsoff D, Summers-Chase D. ICSI as an effective therapy for male factor with antisperm antibodies. Arch Androl 2000; 45(3): 125-30.

[61] Balaban B, Urman B, Isiklar A, et al. Blastocyst transfer following intracytoplasmic injection of ejaculated, epididymal or testicular spermatozoa. Hum Reprod 2001; 16(1): 125-9.

[62] Schlegel PN, Palermo GD, Goldstein M, et al. Testicular sperm extraction with intracytoplasmic sperm injection for nonobstructive azoospermia. Urology 1997; 49(3): 435-40.

[63] Mateizel I, Verheyen G, Van Assche E, Tournaye H, Liebaers I, Van Steirteghem A. FISH analysis of chromosome X, Y and 18 abnormalities in testicular sperm from azoospermic patients. Hum Reprod 2002; 17(9): 2249-57.

[64] Liu CH, Tsao HM, Cheng TC, et al. DNA fragmentation, mitochondrial dysfunction and chromosomal aneuploidy in the spermatozoa of oligoasthenoteratozoospermic males. J Assist Reprod Genet 2004; 21(4): 119-26.

[65] Tesarik J. Paternal effects on cell division in the human preimplantation embryo. Reprod Biomed Online 2005; 10(3): 3705.

[66] La Sala GB, Nicoli A, Capodanno F, Rondini I, Villani MT, Iannotti F. The effect of the 2004 Italian law on outcomes of assisted reproduction technology in severe male factor infertility. Reprod Biomed Online 2010; 20(1): 2-10.

[67] Verza Jr S, Schneider DT, Esteves SC. Limiting the number of oocytes to be fertilized in vitro can dramatically decrease the ability of men with severe male factor infertility to have children [abstract]. Fertil Steril 2007; 88(Suppl 1): 371.

[68] Mostafa T, Anis TH, El-Nashar A, Imam H, Othman IA. Varicocelectomy reduces reactive oxygen species levels and increases antioxidant activity of seminal plasma from infertile men with varicocele. Int J Androl 2001; 24(5): 261-5.

[69] Zini A, Blumenfeld A, Libman J, Willis J. Beneficial effect of microsurgical varicocelectomy on human sperm DNA integrity. Hum Reprod 2005; 20(4): 1018-21.

[70] Esteves SC, Glina S. Recovery of spermatogenesis after microsurgical subinguinal varicocele repair in azoospermic men based on testicular histology. Int Braz J Urol 2005; 31(6): 541-8.

[71] Ficarra V, Cerruto MA, Liguori G, et al. Treatment of varicocele in subfertile men: The Cochrane Review--a contrary opinion. Eur Urol 2006; 49(2): 258-63.

[72] Matthews GJ, Matthews ED, Goldstein M. Induction of spermatogenesis and achievement of pregnancy after microsurgical varicocelectomy in men with azoospermia and severe oligoasthenospermia. Fertil Steril 1998; 70(1): 71-5.

[73] Esteves SC, Oliveira FV, Bertolla RP. Clinical outcome of intracytoplasmic sperm injection in infertile men with treated and untreated clinical varicocele. J Urol 2010; 184(4): 1442-6.

[74] Esteves SC, Verza Jr S, Prudencio C, Seoul B. Success of percutaneous sperm retrieval and intracytoplasmic sperm injection (ICSI) in obstructive azoospermic (OA) men according to the cause of obstruction [abstract]. Fertil Steril 2010; 94(Suppl 4): 233.

[75] Esteves SC, Verza Jr S, Prudencio C, Seol B. Sperm retrieval rates (SRR) in nonobstructive azoospermia (NOA) are related to testicular histopathology results but not to the etiology of azoospermia [abstract]. Fertil Steril 2010; 94(Suppl 4): 132.

[76] Prudencio C, Seol B, Esteves SC. Reproductive potential of azoospermic men undergoing intracytoplasmic sperm injection is dependent on the type of azoospermia [abstract]. Fertil Steril 2010; 94(Suppl 4): 232-3.

[77] Society for Assisted Reproductive Technology and the American Society for Reproductive Medicine. Assisted reproductive technology in the United States: 2001 results generated from the American Society for Reproductive Medicine/ Society for Assisted Reproductive Technology registry. Fertil Steril 2007; 87(6): 125366.

[78] de Mouzon J, Goossens V, Bhattacharya S. Assisted reproductive technology in Europe, 2006: results generated from European registers by ESHRE. Hum Reprod 2010; 25(8): 1851-62.

[79] Latin American Register 2008. Available from: http://www. redlara.com/ing/reg 2008.asp. [cited November 30th, 2010].

[80] Ge SQ, Kang XJ, Duan F. The genetic and epigenetic defect from the sperm for intracytoplasmic sperm injection (ICSI). Yi Chuan 2010; 32(4): 289-94. 
[81] Faure AK, Aknin-Seifer I, Frérot G, et al. Predictive factors for an increased risk of sperm aneuploidies in oligo-asthenoteratozoospermic males. Int J Androl 2007; 30(3): 153-62.

[82] Inci $\mathrm{K}$, Hascicek $\mathrm{M}$, Kara $\mathrm{O}$, et al. Sperm retrieval and intracytoplasmic sperm injection in men with nonobstructive azoospermia, and treated and untreated varicocele. J Urol 2009; 182(4): 1500-5.

[83] Weedin JW, Khera M, Lipshultz LI. Varicocele repair in patients with nonobstructive azoospermia: A meta-analysis. J Urol 2010; 183(6): 2309-15.

[84] Nicopoullos JD, Gilling-Smith C, Almeida PA, Norman-Taylor J, Grace I, Ramsay JW. Use of surgical sperm retrieval in azoospermic men: a meta-analysis. Fertil Steril 2004; 82(3): 691701.

[85] Friedler S, Raziel A, Strassburger D, Soffer Y, Komarovsky D, Ron-El R. Testicular sperm retrieval by percutaneous fine needle aspiration compared to testicular sperm extraction by open biopsy in men with non-obstructive azoospermia. Hum Reprod 1997; 12(7): 1488-93.

[86] Esteves SC, Verza Jr S, Gomes AP. Successful retrieval of testicular spermatozoa by microdissection (micro-TESE) in nonobstructive azoospermia is related to testicular histology [abstract]. Fertil Steril 2006; 86(Suppl 1): 354.

[87] Sousa M, Cremades N, Silva J, et al. Predictive value of testicular histology in secretory azoospermic subgroups and clinical outcome after microinjection of fresh and frozen-thawed sperm and spermatids. Hum Reprod 2002; 17(7): 1800-10.

[88] Fasouliotis SJ, Safran A, Porat-Katz A, Simon A, Laufer N, Lewin A. A high predictive value of the first testicular fine needle aspiration in patients with non-obstructive azoospermia for sperm recovery at the subsequent attempt. Hum Reprod 2002; 17(1): 13942.

[89] Donoso P, Tournaye H, Devroey P. Which is the best sperm retrieval technique for non-obstructive azoospermia? A systematic review. Hum Reprod Update 2007; 13(6): 539-49.
[90] Okada H, Dobashi M, Yamazaki T, et al. Conventional versus microdissection testicular sperm extraction for nonobstructive azoospermia. J Urol 2002; 168(3): 1063-7.

[91] Tsujimura A, Matsumiya K, Miyagawa Y, et al. Conventional multiple or microdissection testicular sperm extraction: a comparative study. Hum Reprod 2002; 17(11): 2924-9.

[92] Schlegel PN. Testicular sperm extraction: microdissection improves sperm yield with minimal tissue excision. Hum Reprod 1999; 14(1): 131-5.

[93] Ramasamy R, Lin K, Gosden LV, Rosenwaks Z, Palermo GD, Schlegel PN. High serum FSH levels in men with nonobstructive azoospermia does not affect success of microdissection testicular sperm extraction. Fertil Steril 2009; 92(2): 590-3.

[94] Schlegel PN, Su LM. Physiological consequences of testicular sperm extraction. Hum Reprod 1997; 12(8): 1688-92.

[95] Alukal JP, Lamb DJ. Intracytoplasmic sperm injection (ICSI)what are the risks. Urol Clin North Am 2008; 35(2): 277-88.

[96] Knoester M, Helmerhorst FM, Vandenbroucke JP, et al. Cognitive development of singletons born after intracytoplasmic sperm injection compared with in vitro fertilization and natural conception. Fertil Steril 2008; 90(2): 289-96.

[97] Belva F, Henriet S, Liebaers I, et al. Medical outcome of 8-yearold singleton ICSI children and a spontaneously conceived comparison group. Hum Reprod 2007; 22(2): 506-15.

[98] Woldringh GH, Besselink DE, Tillema AH, Hendriks JC, Kremer JA. Karyotyping, congenital anomalies and follow-up of children after intracytoplasmic sperm injection with non-ejaculated sperm: a systematic review. Hum Reprod Update 2010; 16(1): 12-9.

[99] Ciriminna R, Papale ML, Artini PG, et al. Impact of Italian legislation regulating assisted reproduction techniques on ICSI outcomes in severe male factor infertility: a multicentric survey. Hum Reprod 2007; 22(9): 2481-7.

(C) Esteves and Schneider; Licensee Bentham Open.

This is an open access article licensed under the terms of the Creative Commons Attribution Non-Commercial License (http://creativecommons.org/licenses/by$\mathrm{nc} / 3.0 /$ ), which permits unrestricted, non-commercial use, distribution and reproduction in any medium, provided the work is properly cited. 